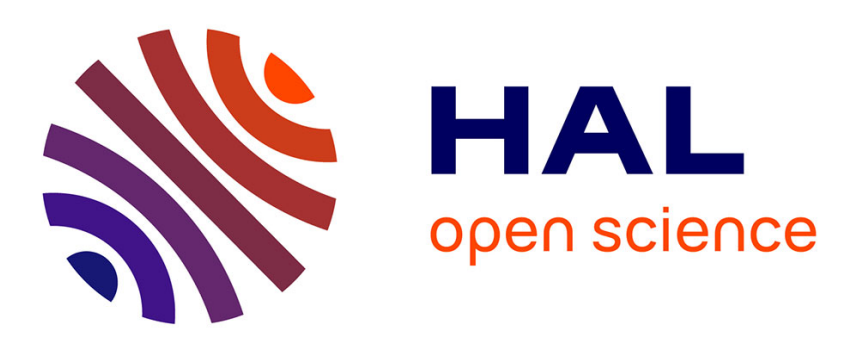

\title{
Design of a continuously tunable delay line using vectorial modulational instability and chromatic dispersion in optical fibers
}

Stéphane Pitois, Christophe Finot, Julien Fatome, Guy Millot

\section{To cite this version:}

Stéphane Pitois, Christophe Finot, Julien Fatome, Guy Millot. Design of a continuously tunable delay line using vectorial modulational instability and chromatic dispersion in optical fibers. Optics Communications, 2009, 282 (5), pp.1016-1019. 10.1016/j.optcom.2008.11.071 . hal-00408622

\section{HAL Id: hal-00408622 \\ https://hal.science/hal-00408622}

Submitted on 15 Apr 2010

HAL is a multi-disciplinary open access archive for the deposit and dissemination of scientific research documents, whether they are published or not. The documents may come from teaching and research institutions in France or abroad, or from public or private research centers.
L'archive ouverte pluridisciplinaire HAL, est destinée au dépôt et à la diffusion de documents scientifiques de niveau recherche, publiés ou non, émanant des établissements d'enseignement et de recherche français ou étrangers, des laboratoires publics ou privés. 


\title{
Design of a continuously tunable delay line using vectorial modulational instability and chromatic dispersion in optical
}

\section{fibers}

\author{
S. Pitois, C. Finot, J. Fatome and G. Millot \\ Institut Carnot de Bourgogne (ICB) UMR 5209 CNRS/Université de Bourgogne, \\ 9 av. Alain Savary, 21078 Dijon, France \\ Authore-mail address : Guy.Millot@u-bourgogne.fr
}

\begin{abstract}
We design an all-optical tunable delay line based on both dispersive and wavelength conversion stages involving modulational instability of a twofrequency pump field propagating in a highly birefringent fiber. More precisely, we numerically show that, by varying the frequency separation between the two orthogonally polarized pump waves, we achieve a controllable and continuous delay of hundreds of picoseconds for signal pulse durations from picoseconds to nanoseconds, without pulse distortion and with only small peak power fluctuations. The proposed method does not require any tunable bandpass filter and can be applied to delay digital data streams at tens of Gbit/s depending on the pump power level.
\end{abstract}




\section{I - INTRODUCTION}

Over the last few years, numerous methods have been developed to satisfy the need for optically tunable pulse delay lines involved in various applications including optical buffering, routers and packet synchronization [1]. Researches on slow light or tunable delay lines are currently focused on the design of all-optical devices which are compatible with high-speed fiber-optic communication technologies. Several demonstrations of controlling the group velocity of light pulses in optical fibers have been reported using either gain-dependent variation of the refractive index or wavelength conversion followed by dispersion. Slow-light delay lines based on a variation of the refractive index associated with a gain have been demonstrated via stimulated Brillouin scattering (SBS) [2,3], stimulated Raman scattering (SRS) [4], optical parametric amplification (OPA) [5] and Raman assisted parametric amplification [6]. An alternative strategy consists of exploiting the spectral variation of the group velocity of an optical fiber by combining wavelength conversion, group-velocity dispersion and wavelength reconversion. More explicitly, in a first stage, the signal beam is converted into a different wavelength by means of a nonlinear process. Then, in a second stage, the frequency-shifted wave travels through a strongly dispersive medium so as to undergo a time delay and finally, the time-shifted beam is subsequently frequency shifted back to the original wavelength by means of another similar nonlinear process. The last few years, several demonstrations of this hybrid technique have been proposed based on various nonlinear processes involving self-phase modulation (SPM) [7] or multiple-wave mixing approaches [8-15]. In particular, differential signal delays above 100 ns have been obtained very recently in optical delay lines based on wavelength conversion taking place in highly nonlinear fibers (HNLF) [11] or periodically-poled Lithium Niobate (PPLN) [12]. Other original schemes have also been proposed which take benefit of the peculiar features of optical soliton collision 
[16] as well as soliton self-frequency shift phenomenon (SSFS) [17]. Generally, all the above mentioned techniques present advantages and limitations which depend on the features of the initial signal pulses to be delayed (duration, repetition rate, power or wavelength...).

In this paper, we present a novel method for generating variable large delays based on the hybrid technique of wavelength conversion and dispersion, where the nonlinear wavelength conversion is achieved via the modulational instability (MI) of two orthogonally polarized pump waves with different frequencies copropagating in a polarization maintaining fiber (PMF) [1820]. More precisely, we theoretically show that delays could be obtained over a wide range by simply adjusting the wavelength separation between the two pumps. The remarkable features of this new tunable delay line (TDL) are its compatibility with both short (ps) or long (ns) pulses and the small fluctuations of the output peak power. Furthermore, we have numerically evaluated our scheme in terms of system performances by monitoring the eye-diagram of a delayed 40Gbit/s optical signal.

\section{II - MODULATIONAL INSTABILITY IN A HIGHLY BIREFRINGENT FIBER}

Throughout this work and as shown in Fig. 1, we consider a lossless fiber with a strong intrinsic birefringence, in which two waves vibrating at different angular frequencies $\omega_{\mathrm{p}}$ and $\omega_{\mathrm{q}}$, polarized respectively along the fast $(\mathrm{x})$ and slow (y) birefringence axis, copropagate along the $z$ axis in the normal dispersion regime. Under the usual slowly-varying envelope approximation, the amplitudes $E_{p}$ and $E_{q}$ of the two electric fields are found to satisfy the following set of coupled nonlinear Schrödinger (NLS) equations [21] : 


$$
\begin{aligned}
& \frac{\partial E_{p}}{\partial z}-\frac{\delta}{2} \frac{\partial E_{p}}{\partial t}+\frac{i}{2} \beta_{2} \frac{\partial^{2} E_{p}}{\partial t^{2}}=i \gamma\left(\left|E_{p}\right|^{2}+\frac{2}{3}\left|E_{q}\right|^{2}\right) E_{p} \\
& \frac{\partial E_{q}}{\partial z}+\frac{\delta}{2} \frac{\partial E_{q}}{\partial t}+\frac{i}{2} \beta_{2} \frac{\partial^{2} E_{q}}{\partial t^{2}}=i \gamma\left(\left|E_{q}\right|^{2}+\frac{2}{3}\left|E_{p}\right|^{2}\right) E_{q},
\end{aligned}
$$

where $\beta_{2}$ is the group-velocity dispersion (GVD) coefficient, which is assumed to be the same value along the two axes for small frequency separations $\omega_{\mathrm{q}}-\omega_{\mathrm{p}}=\Delta \omega$, as considered in this work. $\gamma$ designates the nonlinear Kerr coefficient and $\delta$ the group-velocity mismatch (GVM) of the two waves defined as:

$$
\delta=\delta_{0}+\Delta \omega \beta_{2}
$$

where $\delta_{0}=\Delta \mathrm{n} / \mathrm{c}$ is the GVM in the case of two identical frequencies $(\Delta \omega=0)$ and $\Delta \mathrm{n}$ designates the intrinsic birefringence of the fiber, whose variation as a function of the frequency is supposed to be negligible. As shown by Eq. (2), GVM depends, through chromatic dispersion, on the frequency separation between the two pumps.

Modulational instability in Eqs. (1) is examined by a standard linear-stability analysis applied to the steady state solutions of the NLS equations and yields to a fourth-order dispersion relation that provides the MI power gain spectrum $\mathrm{G}(\Omega)$, where $\Omega$ is the MI frequency [19]. For the sake of simplicity, both pump waves are assumed to propagate with the same power P. In addition, we consider that GVM is sufficiently large so that the input power level $\mathrm{P}$ is always much lower than the critical power $\mathrm{P}_{\mathrm{c}}$ beyond which $\mathrm{MI}$ vanishes [20]. Under these conditions only two MI sidebands can exist, a Stokes signal wave $\omega_{\mathrm{s}}$ and an anti-Stokes idler wave $\omega_{\mathrm{i}}$ polarized along the slow and fast axis, respectively (Fig. 1). 
Figure 2(a) shows the gain spectrum obtained for different input pump powers $\mathrm{P}$ when $\Delta \omega=0$ with the following fiber parameters: intrinsic group-velocity mismatch $\delta_{\mathrm{o}}=0.5 \mathrm{ps} / \mathrm{m}$, nonlinear coefficient $\gamma=0.01 \mathrm{~m}^{-1} \mathrm{~W}^{-1}$ and GVD coefficient $\beta_{2}=0.02 \mathrm{ps}^{2} \mathrm{~m}^{-1}$. For each input power, we observe that the MI gain extends over a narrow band whose width is given by [22] :

$$
\Delta \Omega=\sqrt{\frac{\delta^{2}}{\beta_{2}^{2}}-\frac{20}{3} \frac{\gamma P}{\beta_{2}}}-\sqrt{\frac{\delta^{2}}{\beta_{2}^{2}}-\frac{4}{3} \frac{\gamma P}{\beta_{2}}} .
$$

The maximum MI gain occurs at the optimum frequency $\Omega_{\text {opt }}$ whose a good approximation could be obtained by means of the phase-matching conditions of the four-wave-mixing process responsible for MI [20]:

$$
\Omega_{\text {opt }}=\frac{\delta+\sqrt{\delta^{2}-8 \beta_{2} \gamma P}}{2 \beta_{2}} .
$$

We also see in Fig. 2(a) that in the case of $\Delta \omega=0$, both the MI frequency bandwidth and the maximum MI gain dramatically increase as a function of input pump power. Figure 2(b) shows the MI gain versus $\omega_{\mathrm{s}}-\omega_{\mathrm{p}}$ for different pump frequency separations and an identical pump power $\mathrm{P}$ of $5 \mathrm{~W}$. Here, the frequency of the pump wave polarized along the slow axis is variable, whereas the frequency of the wave polarized along the fast axis is fixed. As can be seen in Fig. 2(b), variations of the pump frequency separation over a range of $3 \mathrm{THz}$ lead to only small 
fluctuations of the MI peak gain and optimal frequency. Furthermore, Fig. 2(b) shows that, as the GVM increases, the MI bandwidth decreases, as predicted by Eqs. (2) to (4).

Let us consider now that a signal pulse with frequency $\omega_{\mathrm{s}}$ is launched into the PMF and let us assume that it is linearly polarized along the slow birefringence axis. The frequency detuning between the pump wave with a fixed frequency $\omega_{p}$ and the signal pulse is carefully chosen in such a way that the signal frequency falls within the MI-gain sideband. More precisely, this frequency detuning is chosen close to the MI frequency for $\Delta \omega=0$ and can be obtained from Eqs. (2) and (4):

$$
\omega_{p}-\omega_{s}=\Omega_{o p t}(\Delta \omega=0)=\Omega_{o p t}^{o}=\frac{\delta_{o}+\sqrt{\delta_{o}^{2}-8 \beta_{2} \gamma P}}{2 \beta_{2}}
$$

As shown in Fig. 2(b), in presence of the second pump wave $\omega_{\mathrm{q}}$, for variations of $\Delta \omega$ on both sides of $\Delta \omega=0$, the signal remains close to the MI peak gain. As a consequence, the signal amplification by the MI process will be only slightly affected by the variations of $\Delta \omega$. Under these conditions, a strong frequency conversion occurs via MI and an idler conjugated pulse is generated and amplified at a frequency $\omega_{i}=\omega_{q}+\Omega_{o p t}^{o}$. The most important result here is that the frequency detuning between the idler and signal pulses is simply given by :

$$
\delta \omega=\Delta \omega+2 \Omega_{o p t}^{o} .
$$

\section{III - DESIGN OF A LONG TUNABLE DELAY LINE}


The proposed delay generator is based on the principle proposed in [8,9]: first, the signal wavelength is frequency converted in a controllable way and the resulting idler signal is timedelayed through propagation in a dispersive fiber. The wavelength of the time-delayed idler signal is finally frequency shifted back to its initial wavelength. In the scheme presented in this paper, the wavelength conversion stage is ensured by vectorial MI of two pump waves with different frequencies copropagating in a PMF. The variation of delay experienced by the signal is thus proportional to the frequency detuning $\delta \omega$ as well as to the GVD coefficient $\beta_{2}^{\prime}$ and length $L^{\prime}$ of the dispersive fiber. As shown by Eq. (6), $\delta \omega$ can be easily tuned over a wide range by simply adjusting the frequency separation $\Delta \omega$ between the two pump waves. $\Delta \omega$ acts thus as a practical control parameter for the signal pulse delay. The variation of the signal pulse's delay with respect to the case $\Delta \omega=0$ (single frequency regime) is simply given by:

$$
\Delta \tau=\beta_{2}^{\prime} L^{\prime} \Delta \omega
$$

In Fig. 3, we propose a schematic diagram of such a tunable optical delay line. The pump wave $\omega_{\mathrm{q}}$ and the signal pulses $\omega_{\mathrm{s}}$ are combined by a fiber polarization-maintaining (PM) 90:10 coupler. Their polarization states are aligned parallel. The pump $\omega_{\mathrm{q}}$ and the signal $\omega_{\mathrm{s}}$ are then combined with the second pump $\omega_{\mathrm{p}}$ by means of a fiber polarization combiner. The three beams are then injected into a highly-nonlinear polarization-maintaining fiber (HNL-PMF). The pump wave $\omega_{\mathrm{p}}$ is polarized along the fast axis, whereas the two other waves are polarized along the slow axis. At the output of the HNL-PMF, a polarizer aligned along the fast axis rejects the signal pulse and the tunable pump $\omega_{\mathrm{q}}$. On the other hand, a large bandwidth optical bandpass filter (OBPF 1) rejects the fixed pump $\omega_{\mathrm{p}}$ so as to select the idler wave $\omega_{\mathrm{i}}$. The idler pulse propagates through a span of 
dispersive standard single-mode fiber (SMF) to undergo the time delay. Similarly to the previous stage, the time-shifted idler pulse is then spectrally shifted back to the original signal wavelength via MI which takes place in a second identical HNL-PMF pumped by both waves $\omega_{\mathrm{p}}$ and $\omega_{\mathrm{q}}$. Finally, the two pump waves and the idler pulse are filtered from the delayed signal pulse by means of a polarizer aligned along the slow axis coupled with an optical bandpass filter (OBPF 2) centered on the signal wavelength. Let us note that a second identical span of SMF can be used to compensate for dispersion pulse broadening experienced in the first span of SMF in the case where short input signal pulses are delayed. It is also worth noting that since the idler and signal pulses are phase conjugated via the MI process, the chirp profile is reversed during the wavelength back-conversion so that it can be compensated by the same optical fiber than the one used to delay the idler pulse.

Figure 4(a) shows the intensity profiles of the delayed pulses versus frequency detuning in the case of an input signal pulse having a full width at half maximum (FWHM) of 25 ps and a peak power $\mathrm{P}_{\mathrm{s}}$ of $10 \mathrm{~mW}$. These results were obtained from numerical simulations of Eqs. (1) with two pump waves of identical peak powers $\mathrm{P}$ of $5 \mathrm{~W}$. The frequency separation $\omega_{\mathrm{p}}-\omega_{\mathrm{s}}$ between the fixed pump and the signal is $3.93 \mathrm{THz}$. The HNL-PMF length for achievement of efficient wavelength conversion was found to be $\mathrm{L}=27 \mathrm{~m}$. Under the above conditions, the idler pulse generated at the fiber output has a peak power of $12 \mathrm{~mW}$. The dispersive SMF has a GVD coefficient $\beta_{2}^{\prime}$ of $0.02 \mathrm{ps}^{2} / \mathrm{m}$ and a length $\mathrm{L}^{\prime}$ of $1000 \mathrm{~m}$. After wavelength reconversion of the idler pulse, the delayed signal pulse is filtered with an optical bandpass filter (OBPF 2). As shown in Fig. 4(a), after passing through the delay line, the signal pulse has preserved its duration and profile. Another noticeable feature in Fig. 4(a) is that the peak power of the delayed signal 
remains nearly constant for a variation of $\Delta \omega$ of $3 \mathrm{THz}$, corresponding to a variation of the delay $\Delta \tau$ of nearly $380 \mathrm{ps}$. Figure 4(b) shows similar results obtained for a shorter signal pulse of $6.5 \mathrm{ps}$ and with the following parameters: $\mathrm{P}=20 \mathrm{~W}, \omega_{\mathrm{p}}-\omega_{\mathrm{s}}=3.83 \mathrm{THz}$ and $\mathrm{L}=7 \mathrm{~m}$. Note that pulse broadening which occurs in the SMF in that case is compensated with a second identical SMF. The outstanding features in Fig. 4(b) are that, similarly to the case of the 25-ps signal pulse, the 6.5-ps pulse has preserved its duration and intensity profile, and secondly the peak power variation is rather small.

To study in more details the system performances of the delay generator, we have performed numerical simulations with VPI WDM transmission maker software by considering a degraded 40-Gbit/s pseudo-random bit sequence (PRBS) of 128-bits (after, for example propagation in a usual transmission line). The other parameters are the same as in Fig. 4(a). Figure 5 represents the simulated eye diagrams for the transmission signal as well as the delayed signal pulse train for three different pump separations $\Delta \omega$. We clearly see from Fig. 5 that both the eye opening and the magnitude of the amplitude and timing jitters are not modified by the delay generator.

Finally, we would like to emphasize that longer delay could be obtained simply by increasing the length of the dispersive fiber (SMF). As pointed out in Ref. [13], the maximum delay value that can be realized is then essentially limited by the residual dispersion originating from the thirdorder dispersion of the SMF fiber, so that more than $100 \mathrm{~ns}$ delay should be obtained for 10 Gbit/s pulses trains. 


\section{IV - CONCLUSIONS}

In this work, we have numerically demonstrated the efficiency of a continuously tunable delay generator based on vectorial modulational instability of two pump frequencies in a highly birefringent fiber. The proposed technique is applicable to a wide range of pulse durations and delays without any significant distortion and peak power variation of the signal pulses. Moreover, this approach is not restricted to a specific modulation format and is phase preserving so that it could be also suitable for phase-modulated signal. Another interesting point is that the method presented in this paper does not need any bandpass filter tuned to different centered wavelengths but only used bandpass filters centered at fixed wavelengths. Finally, the power level necessary to obtain a wide bandwith is on the same order of magnitude as for methods using Raman amplification $[3,6]$ but remains about one order of magnitude higher than those required in techniques based on Brillouin or dual-pump parametric amplifiers [2,13]. Nevertheless, this power could be significantly reduced by using more nonlinear HNLF, such as a photonic crystal fiber. We believe that these results may be relevant for various applications including optical buffering in telecommunication systems. 


\section{V - REFERENCES}

1. A.E. Willner, B. Zhang, L. Zhang, L.Yan and I. Fazal, "Optical signal Processing using tunable delay elements based on slow light,” IEEE J. Select. Top. Quant. Electron. 14, 691-705 (2008).

2. K. Y. Song, M. G. Herraez, and L. Thévenaz, "Observation of pulse delaying and advancement in optical fibers using stimulated Brillouin scattering," Opt. Express 13, 82-88 (2005).

3. Y. Okawachi, M. S. Bigelow, J. E. Sharping, Z. Zhu, A. Schweinsberg, D. J. Gauthier, R. W. Boyd, and A. L. Gaeta, "Tunable all-optical delays via Brillouin slow light in an optical fiber," Phys. Rev. Lett. 94, 153902 1-4 (2005).

4. J. E. Sharping, Y. Okawachi, and A. L. Gaeta, "Wide bandwidth slow light using a Raman fiber amplifier," Opt. Express 13, 6092-6098 (2005).

5. L. Yi, W. Hu, Y. Su, M. Gao, and L. Leng, "Design and system demonstration of a tunable slow light delay line based on fiber parametric process," IEEE Photon. Technol. Lett. 18, 2575-2577 (2006).

6. D. Dahan and G. Eisenstein, "Tunable all optical delay via slow and fast light propagation in a Raman assisted fiber optical parametric amplifier: a route to all optical buffering," Opt. Express 13, 6234-6249 (2005).

7. Y. Okawachi, J. E. Sharping, C. Xu, and A. L. Gaeta, "Large tunable optical delays via self-phase modulation and dispersion," Opt. Express 14, 12022-12027 (2006).

8. M. Burzio, P. Cinato, R. Finotti, P. Gambini, M. Puleo, E. Vezzoni, L. Zuchelli, "Optical Cell Synchronisation in an ATM Switch”, Proc. ECOC 1994, 581-584 (1994).

9. J. E. Sharping, Y. Okawachi, J. van Howe, C. Xu, Y. Wang, A. E. Willner, and A. L. Gaeta, "Alloptical, wavelength and bandwidth preserving, pulse delay based on parametric wavelength conversion and dispersion," Opt. Express 13, $7872-7877$ (2005).

10. Irfan Fazal, Omer Yilmaz, Scott Nuccio, Bo Zhang, Alan E. Willner, Carsten Langrock and Martin M. Fejer, "Optical data packet synchronization and multiplexing using a tunable optical delay based on wavelength conversion and inter-channel chromatic dispersion”, Opt. Express 15, 1049210497 (2007).

11. N. Alic, J.R. Windmiller, J.B. Coles, S. Moro, E. Myslivets, R.E. Saperstein, J.M. Chavez Boggio, C.S. Bres and S. Radic, "105-ns Continuously Tunable Delay of 10-Gb/s Optical Signal”, IEEE Photon. Technol. Lett. 20, 1187-1189 (2008). 
12. L. Christen, I. Fazal, O.F. Yilmaz, X. Wu, S. Nuccio, A.E. Willner, C. Langrock, M.M. Fejer, "Tunable 105-ns Optical Delay for 80-Gbit/s Rz-DQPSK, 40-Gbit/s RZ-DPSK, and 40-Gbit/s RZOOK Signals using Wavelength Conversion and Chromatic Dispersion”, OFC/NFOEC 2008, OTuD1 (2008).

13. Nikola Alic, Joshua Ray Windmiller, James B. Coles and Stojan Radic, "Two-Pump Parametric Optical Delays”, IEEE J. of Select. Top. Quant. Electron. 14, 681-690 (2008).

14. Mable P.Fok and Chester Su, "Tunable Optical Delay Using Four-Wave Mixing in a 35-cm Highly Nonlinear Bismuth-Oxide Fiber and Group Velocity Dispersion”, J. of Light. Technol. 26, 499-504 (2008).

15. C.J. McKinstrie, S. Radic and A.R. Chraplyvy, "Parametric Amplifiers Driven by Two Pump Waves", IEEE J. of Select. Top. Quant. Electron. 8, 538-547 (2002).

16. T. Kunihiro, A. Maeda, S. Oda, and A. Maruta, "Experimental demonstration of all-optical tunable delay line based on distortion-less slow and fast light using soliton collision in optical fiber," Opt. Express 14, 11736-11747 (2006).

17. S. Oda and A. Maruta, "All-optical tunable delay line based on soliton self-frequency shift and filtering broadened spectrum due to self-phase modulation,” Opt. Express 14, 7895-7902 (2006).

18. P.D. Drummond, T.A.B. Kennedy, J.M. Dudley, R. Leonhardt and J.D. Harvey, "Cross-phase modulationnal instability in high-birefringence fibers", Opt. Commun. 78, 137-142 (1990).

19. E. Seve, P. Tchofo Dinda, G. Millot, M. Remoissenet, J.M. Bilbault, and M. Haelterman, "Modulational instability and critical regime in a highly birefringent fiber," Phys. Rev. A 54, 35193534 (1996).

20. P. Tchofo Dinda, G. Millot, E. Seve, and M. Haelterman, "Demonstration of a nonlinear gap in the modulational instability spectra of wave propagation in highly birefringent fibers," Opt. Lett. 54, 3519-3534 (1996).

21. G.P. Agrawal, "Nonlinear Fiber Optics", Third Edition, Academic Press (2001).

22. C.J. McKintrie, S. Radic and C. Xie, "Parametric instabilities driven by orthogonal pump waves in birefringent fibers", Opt. Express 11, 2619-2633 (2003) 


\section{Figure Captions}

Fig. 1. Principle of the method: Schematic diagram of the four waves propagating through the highly birefringent fiber.

Fig. 2. (a) MI gain versus sideband MI frequency detuning for different input pump powers and $\Delta \omega=0$. (b) MI gain versus $\left(\omega_{\mathrm{s}}-\omega_{\mathrm{p}}\right)$ for different frequency separations $\Delta \omega$ and $\mathrm{P}=5 \mathrm{~W}$.

Fig. 3. Schematic diagram of the continuously optical delay generator

Fig. 4. Numerical traces of the pulses at the delay generator input (inset) and output for $\Delta \omega=-$ $1.5 \mathrm{THz}$ (dashed line), $\Delta \omega=0$ (solid line) and $\Delta \omega=1.5 \mathrm{THz}$ (circles) for : (a) 25-ps pulse width and 5-W pump and (b) 6.5-ps pulse width and 20-W pump power.

Fig. 5. Eye diagrams for a 40-Gbit/s PRBS signal. Pulse train (a) after propagation through a transmission line, at the delay generator output for (b) $\Delta \omega=-1.5 \mathrm{THz}$, (c) $\Delta \omega=0$ and (d) $\Delta \omega=$ $1.5 \mathrm{THz}$. 


\section{S. Pitois et al. Fig. 1.}

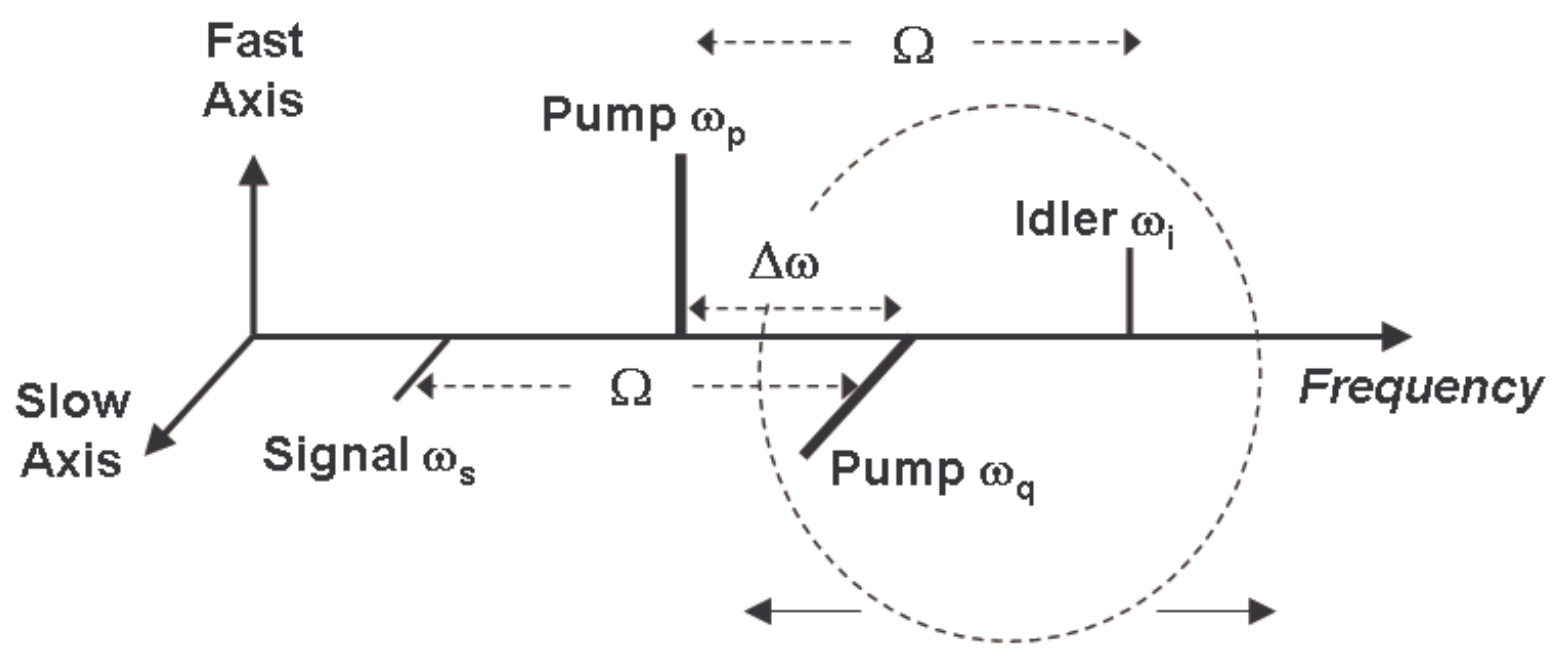

Fig. 1. Principle of the method: Schematic diagram of the four waves propagating through the highly birefringent fiber. 


\section{S. Pitois et al. Fig. 2.}
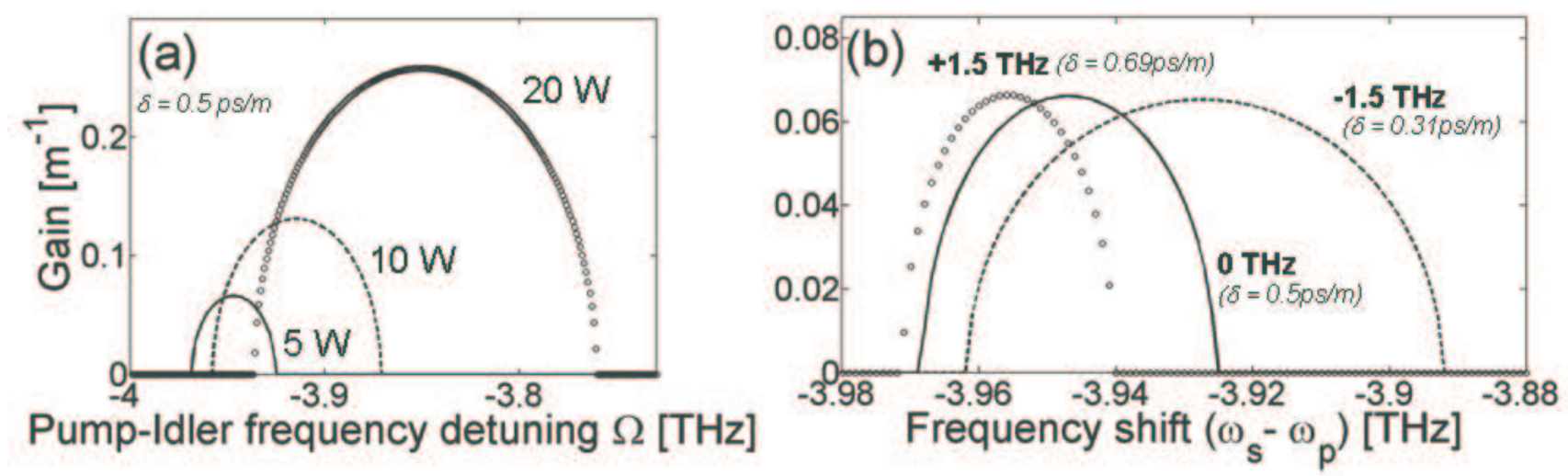

Fig. 2. (a) MI gain versus sideband MI frequency detuning for different input pump powers and $\Delta \omega=0$. (b) MI gain versus $\left(\omega_{\mathrm{s}}-\omega_{\mathrm{p}}\right)$ for different frequency separations $\Delta \omega$ and $\mathrm{P}=5 \mathrm{~W}$. 


\section{S. Pitois et al. Fig. 3.}

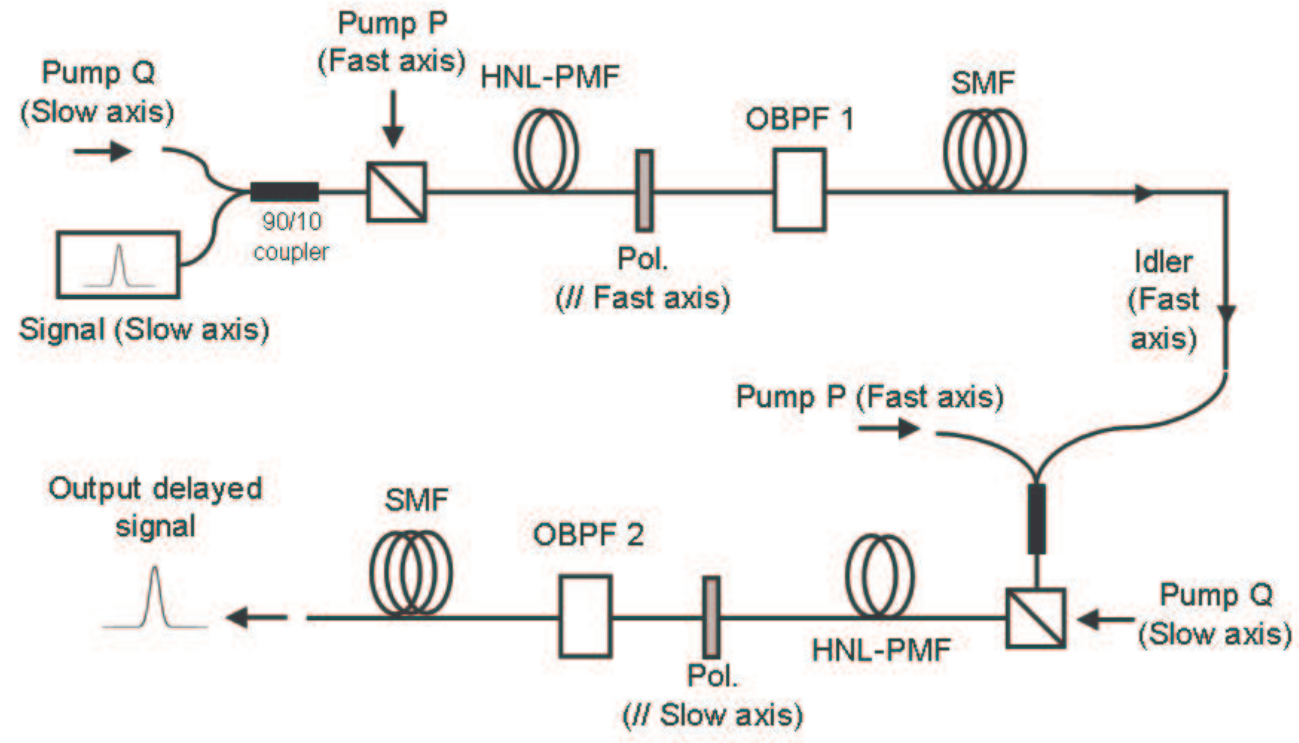

Fig. 3. Schematic diagram of the continuously optical delay generator. 


\section{S. Pitois et al. Fig. 4.}
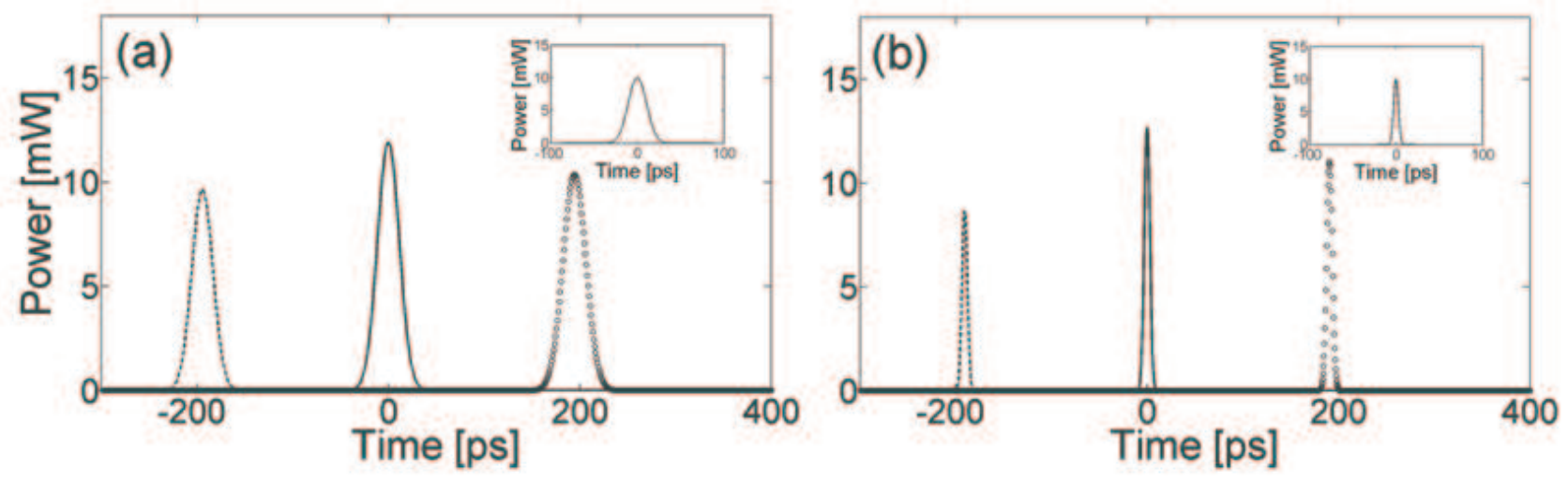

Fig. 4. Numerical traces of the pulses at the delay generator input (inset) and output for $\Delta \omega=-$ $1.5 \mathrm{THz}$ (dashed line), $\Delta \omega=0$ (solid line) and $\Delta \omega=1.5 \mathrm{THz}$ (circles) for : (a) 25-ps pulse width and 5-W pump and (b) 6.5-ps pulse width and 20-W pump power. 


\section{S. Pitois et al. Fig. 5.}

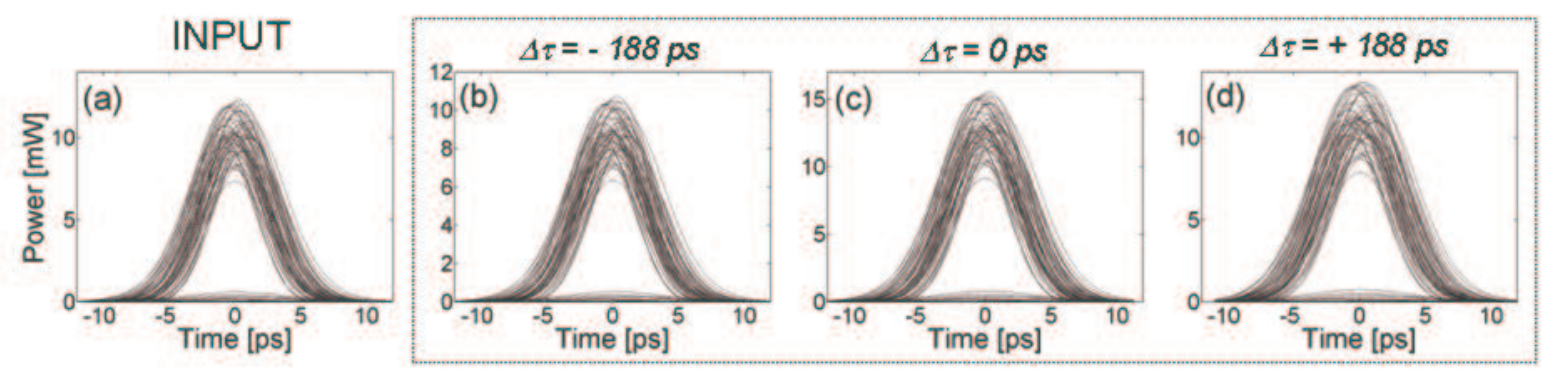

Fig. 5. Eye diagrams for a 40-Gbit/s PRBS signal. Pulse train (a) after propagation through a transmission line, at the delay generator output for (b) $\Delta \omega=-1.5 \mathrm{THz}$, (c) $\Delta \omega=0$ and (d) $\Delta \omega=$ $1.5 \mathrm{THz}$. 